\title{
Diseño de actividades y uso de la coevaluación para fomentar el desarrollo de competencias transversales en ingeniería mecánica y de materiales
}

\author{
J. Carballeira ${ }^{\text {a1 }}$, A. Rovira ${ }^{1}$, J.L. Suñer ${ }^{1}$, E. Nadal ${ }^{1}$, M.J. Rupérez ${ }^{1}$, J. Dols ${ }^{1}$, O. \\ Sahuquillo $^{1}$, J. Martínez-Casas ${ }^{1}$, P. Vila ${ }^{1}$, A. Pedrosa ${ }^{1}$, F.D. Denia ${ }^{1}$, J.J. Ródenas ${ }^{1}$, M. \\ Tur $^{1}$ \\ a(jacarmo@mcm.upv.es)
}

\author{
${ }^{1}$ Departamento de Ingeniería Mecánica y de Materiales
} Universitat Politècnica de València, Camino de Vera s/n, 46022, Valencia

\begin{abstract}
The evaluation activities developed within the frame of a project on innovation and improvement of teaching, and some preliminary results, are presented in this paper. The main goal of this project is to work on the design of evaluation activities that truly force the students to put their soft skills (generic competences) into play and, at the same time, that allow the instructors to grade their students' performance on the technical-scientific competences.An assessment for learning approach is used, so that the evaluation activities are useful for the students to improve their learning experience.
\end{abstract}

Keywords: generic competences, evaluation activities, peer-assessment

\footnotetext{
Resumen

En esta comunicación se presentan las actividades de evaluación desarrolladas en el marco de un Proyecto de Innovación y Mejora Educativa, junto con algunos resultados preliminares. El principal objetivo de este proyecto es el diseño de actividades de evaluación que fuercen a los estudiantes a desarrollar sus competencias transversales, al mismo tiempo que permitan a los profesores evaluar su desempeño en las competencias científico-técnicas. Se emplea un enfoque de evaluación formativa, de forma que las actividades de evaluación sean útiles a los estudiantes para mejorar su aprendizaje.
}

Palabras clave: competencias transversales, actividades de evaluación, coevaluación 


\section{Introducción}

La evaluación de competencias transversales requiere de la puesta en juego de los distintos componentes de la propia competencia: conocer, saber aplicar, y actitudes y valores manifiestos (Villa et al., 2011). De ahí que las actividades de evaluación deban ser más completas que las empleadas tradicionalmente. De las conclusiones y experiencia obtenida tras la aplicación de los resultados de un proyecto de innovación y mejora educativa anterior (Carballeira et al., 2015), se comprobó que un diseño adecuado de las actividades de evaluación resulta clave para mejorar la motivación de los estudiantes y la experiencia de enseñanza-aprendizaje, así como para desarrollar las competencias transversales. Disponer de actividades de evaluación que pongan en juego estas competencias, y al mismo tiempo, sean rigurosas y completas a la hora de evaluar las competencias específicas científico-técnicas, las convierten en herramientas muy útiles para el aprendizaje, y no sólo la calificación de los estudiantes.

En una de las asignaturas involucradas en aquel proyecto se empleó la coevaluación para, en principio, disponer de más calificaciones y poder comprobar la bondad de los instrumentos de evaluación empleados (rúbricas y listas de control) (Carballeira et al. 2016). Cuando se estaban estudiando las respuestas obtenidas por parte de los estudiantes, se planteó que esta coevaluación decía mucho acerca del nivel de dominio del propio estudiante actuando como evaluador.

El empleo de la coevaluación ha sido ampliamente estudiado en la literatura. Son numerosas las referencias en las que se muestra que la aceptación por parte de los estudiantes suele ser favorable (Cavas et al., 2011). Las ventajas que se suelen nombrar son, entre otras (Nulty, 2008; Langan et al., 2003):

- Mejora la motivación de los estudiantes al aumentar su autonomía e incrementar su implicación en la evaluación.

- Favorece que los estudiantes se responsabilicen de su aprendizaje.

- Considera la evaluación como parte del proceso de aprendizaje, de forma que los errores se ven como oportunidades más que como fallos.

- Implica la práctica de competencias genéricas de tipo instrumental, interpersonal y sistemáticas para la evaluación, lo que es especialmente interesante para desarrollar la capacidad de aprendizaje autónomo a largo plazo.

- Proporciona a los estudiantes una realimentación de su propio trabajo desde la perspectiva de otros iguales, lo que mejora su capacidad para autoevaluarse.

- Promueve aprendizajes profundos y no superficiales.

- Aporta un valor de objetividad que propicia el diálogo, revisión y mejora continua del proceso de evaluación.

Entre las desventajas, se mencionan:

- La validez y la consistencia de la coevaluación, de forma que no haya desviaciones en positivo, o en negativo, frente a la heteroevaluación del experto.

(cc) EY-NC-ND 2017, UniversitatPolitècnica de València 
- La inexperiencia de los estudiantes a la hora de evaluar, lo que puede desincentivarles y generar un rechazo por su parte.

Estas desventajas se pueden minimizar siguiendo las recomendaciones habituales para que la coevaluación tenga éxito: simplificar y explicitar al máximo los criterios, formulando los diferentes indicadores en forma de preguntas (Lim, 2003); llevarla a cabo de forma anónima y validar la coherencia con las evaluaciones del profesor (Bostock, 2000).

\section{Objetivos}

Los objetivos de esta innovación han sido dos:

- Por un lado, el diseño y/o mejora de actividades de evaluación en asignaturas del departamento de ingeniería mecánica y de materiales en diferentes títulos y niveles académicos. Se trata de enriquecer el proceso de evaluación de las asignaturas desde el punto de vista formativo, desarrollando para ello actividades más completas que permitan trabajar y evaluar tanto las competencias científicotécnicas, específicas de las materias, como las competencias transversales.

- Por otro lado, en algunas de estas actividades se ha planteado el uso de la coevaluación como herramienta de evaluación adicional, para afinar la calificación de los estudiantes, y mejorar su aprendizaje. La idea es que el análisis de la coevaluación llevada a cabo por un estudiante de otro igual puede ser una fuente de información muy relevante para que el experto pueda evaluar a este mismo estudiante. La forma en que un estudiante puede reconocer y apreciar el dominio de otro igual en una competencia también indica hasta qué punto domina este estudiante la competencia, y además, le puede ayudar a desarrollar la competencia hasta niveles de logro superiores.

En esta comunicación se presentan tres experiencias destacadas, entre las iniciadas en el desarrollo del proyecto de innovación y mejora educativa. Se trata de experiencias relacionadas con las competencias transversales "Diseño y Proyecto", "Compresión e Integración" y "Aplicación y Pensamiento Práctico", siguiendo las denominaciones y definiciones empleadas en el proyecto institucional de la Universitat Politècnica de València, UPV, sobre competencias transversales (UPV, 2014).

\section{Desarrollo de la innovación}

\subsection{Diseño y Proyecto}

La primera experiencia que se va a describir es la propuesta de un trabajo académico para la evaluación y desarrollo de la competencia transversal "Diseño y Proyecto". La experiencia se ha llevado a cabo en la titulación del Máster Universitario en Ingeniería Industrial, en la asignatura Diseño y Aplicación de Equipos Industriales, del primer curso del máster. Participaron alrededor de 90 estudiantes trabajando en equipos de 4. 
El objetivo del trabajo es resolver un problema real en una empresa real. Los estudiantes deben visitar la empresa, detectar problemas relacionados con la logística interna (que es una competencia específica de la asignatura), y proponer soluciones en base al estudio del caso y los conocimientos técnicos adquiridos en la asignatura. Finalmente, deben entregar un informe escrito y realizar una defensa oral de sus conclusiones para el resto de sus compañeros.

La competencia "Diseño y Proyecto" se va a evaluar a través del informe escrito. Los resultados de aprendizaje de esta competencia son:

- Comprobar el contexto del proyecto.

- Plantear los objetivos.

- Establecer un plan de acción para alcanzar los objetivos.

- Obtener realimentación de las acciones y resultados.

- Identificar los posibles riesgos.

Para evaluar estos resultados, la UPV ha desarrollado unas rúbricas según el nivel académico. En este caso, para el nivel de máster, los indicadores asociados a estos resultados son:

1. El estudiante identifica el contexto y las necesidades del proyecto.

2. El estudiante especifica los objetivos del proyecto de forma coherente con las necesidades identificadas en el contexto.

3. El estudiante planifica las acciones de forma eficiente.

4. El estudiante establece herramientas para seguir el desarrollo de las acciones.

5. El estudiante identifica posibles riesgos del proyecto.

6. El estudiante revisa los resultados esperados.

La propuesta es asociar estos indicadores a la evaluación de las diferentes partes del informe escrito, que debe seguir la estructura tradicional de un trabajo académico: Introducción, Desarrollo, Discusión y Conclusiones.

En la Introducción se deben establecer el contexto del problema a resolver, las limitaciones y principales características a tener en cuenta, así como los objetivos; de modo que la calidad de la Introducción en el informe se asocia al nivel de logro en los indicadores 1 y 2.

Siguiendo la misma idea, en el Desarrollo del trabajo se incluyen las acciones y los mecanismos para monitorizar la implementación del plan. Se trata del núcleo de cualquier proyecto. La calidad de esta sección estaría directamente relacionada con los indicadores 3 y 4.

La sección de Discusión suele incluir comentarios acerca de las limitaciones del proyecto, que estarán vinculados a los riesgos del mismo, de forma que se puede asociar al indicador 5. Finalmente, en el apartado de Conclusiones se realiza un análisis de los resultados esperados cuando se pongan en marcha las acciones, por lo que nos proporcionará evidencias para evaluar el indicador 6.

(cc) EY-NC-ND 2017, UniversitatPolitècnica de València 


\subsection{Comprensión e integración}

Esta experiencia se ha llevado a cabo en la titulación del Máster Universitario en Ingeniería del Mantenimiento, en la asignatura Análisis de Vibraciones, y participaron 23 estudiantes.

La actividad de evaluación consistía en la redacción de un trabajo académico y el empleo de la coevaluación para su calificación. Además de los resultados de aprendizaje específicos de la materia, con este trabajo se pretendía desarrollar las competencias en “compresión e integración”, y "comunicación escrita efectiva”. Para la calificación del trabajo, se combinan la evaluación del "experto" (el profesor de la asignatura), y la evaluación entre pares llevada a cabo por los estudiantes. El objetivo de esta experiencia era mejorar la compresión del curso mediante la implicación de los estudiantes en la evaluación de los trabajos de sus compañeros.

El trabajo era realizado en grupos de 2 o 3 estudiantes, o de forma individual. Cada estudiante debía evaluar otro trabajo en el que no hubiese participado, de forma que cada trabajo fue evaluado tantas veces como componentes tuviese el equipo. La nota del trabajo se asignaba a partir de la suma ponderada de la calificación otorgada por el experto y la calificación promediada de la evaluación por pares. En particular, se empleó la siguiente expresión:

$$
\mathrm{C}=0,7 \cdot \mathrm{C}^{\text {experto }}+0,3 \cdot \mathrm{C}^{\text {pares }}
$$

Se empleó una rúbrica con varios indicadores para evaluar el trabajo: la presentación del trabajo escrito; la descripción del modelo; los cálculos; las conclusiones; y el uso adecuado de referencias. La nota obtenida mediante la rúbrica iba de 0 a 9 , ya que se otorgaba un punto extra en función del nivel de autonomía del estudiante en la realización del trabajo.

Si la desviación entre la nota otorgada por el experto y la nota propuesta por el estudianteevaluador era mayor de 3 puntos, se aplicaba un corrección a la nota del evaluador, y para la nota del trabajo evaluado sólo se consideraba la nota del experto.

Los evaluadores fueron asignados de forma aleatoria por el profesor, de forma que los estudiantes no sabían quién o quiénes habían sido sus evaluadores.

\subsection{Aplicación y pensamiento práctico}

Esta experiencia se ha llevado a cabo en el $4^{\circ}$ curso del Grado en Ingeniería en Tecnologías Industriales, en la asignatura Diseño Mecánico Asistido por Ordenador, y participaron 35 estudiantes.

La actividad de evaluación planteada consiste en la redacción de unas instrucciones de montaje de un motor de 4 cilindros en V (ver Fig. 1). Con este trabajo se puede evaluar la competencia Aplicación y Pensamiento Práctico ya que para construir el modelo final no existe una única secuencia de pasos, sino que, utilizando los comandos de edición y gestión de entidades del programa ADAMS/View (ADAMS, 2014), se puede crear el modelo de manera más o menos rápida y con un número de pasos mayor o menor. Estas diferentes vías 
son aplicables tanto a la copia y reubicación de elementos físicos del modelo como a la copia y reprogramación de las funciones de las fuerzas en los pistones.

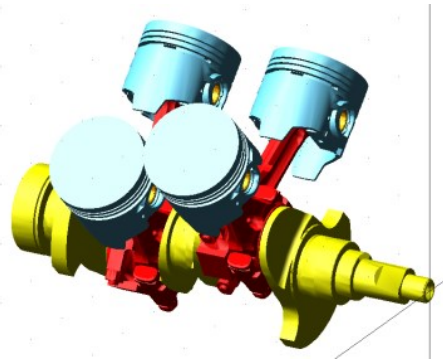

Fig. 1 Motor de 4 cilindros en V modelado mediante ADAMS/View (ADAMS, 2014).

Además, para la creación del motor de 4 cilindros en $\mathrm{V}$, es necesaria la incorporación de elementos nuevos, debido al alargamiento del asiento de las muñequillas del cigüeñal, ya que se calan dos bielas por muñequilla. El modo en que realizan esta modificación se deja por completo a los estudiantes, que pueden hacerla mediante los elementos propios del programa de simulación o con programas externos de CAD, que permiten trabajar con los modelos originales de las piezas.

Dado que en una vez finalizado el modelo del motor policilíndrico puede ser difícil o imposible detectar la secuencia de pasos que han llevado a ese modelo, se pide una memoria del trabajo bajo la forma de un manual de instrucciones, con un formato similar al utilizado por los profesores de la asignatura en sus clases, de manera que cualquier persona con conocimientos del programa y partiendo de la misma situación inicial sea capaz de llegar al mismo modelo que el realizado por los estudiantes. En esas instrucciones se comprobará si los estudiantes alcanzan los resultados de aprendizaje previstos.

Las memorias de los trabajos son evaluadas empleando las dos listas de control mostradas en las Tablas 1 y 2. La lista de control en la Tabla 1 está adaptada a la actividad desarrollada para evaluar la competencia específica, mientras que la lista de la Tabla 2 sirve para evaluar la competencia transversal, pero evitando un lenguaje abstracto, y utilizando uno con más referencias al trabajo realizado.

La evaluación mediante la lista de la Tabla 1 se realiza tanto por parte del profesorado (heteroevaluación) como por parte del alumnado (coevaluación). La nota obtenida se utiliza para calificar la competencia específica, y como una primera aproximación en la evaluación de la competencia transversal, ya que la actividad está basada en su utilización. La valoración final de esta calificación es de $70 \%$ la heteroevaluación y $30 \%$ la coevaluación.

Las preguntas de la lista de control mostrada en la Tabla 2 para evaluar la competencia transversal tienen un valor de 0 a 3 puntos, salvo una cuya casuística no podía tener la misma valoración y tiene una de 0 a 2 puntos.

Las preguntas se dividen en dos bloques. Las 6 primeras hacen hincapié en la evaluación de la competencia transversal. Las 2 últimas están formuladas para que el evaluador de una 
opinión comparativa entre su propio trabajo y el trabajo evaluado. Es importante resaltar que esta opinión es útil para confirmar si el evaluador, partiendo de un nivel de asimilación y comprensión de la competencia transversal, es capaz de reconocerla en el evaluado. En el caso de que el nivel del evaluador esté en tramos bajos, si el nivel del evaluado es alto, la coevaluación le puede poner en contacto con la competencia a ese nivel más alto. En este caso, si la puntuación en estas dos preguntas es alta, se tiene un indicio de que el dominio del evaluador en esta competencia ha aumentado.

El primer bloque de 6 preguntas da una puntuación máxima de 17 puntos, tal y como está diseñada. El segundo bloque da una puntuación máxima de 6 , pero puede hacerse una utilización menos numérica y más de apreciación por parte del profesorado.

La utilización de esta lista para evaluar la competencia transversal es la siguiente: a todos los estudiantes o equipos se les hace una heteroevaluación (experto), la cual se tomará como referencia principal. Por otro lado, un estudiante o equipo evaluador, A, evalúa a otro estudiante o equipo, B, que, se recuerda, ya ha sido evaluado por el profesorado. Tomando la heteroevaluación de B como referencia, se contrastará la coevaluación de A sobre B para determinar si A domina y tiene asimiladas las competencias específicas y transversales. Dicho de otro modo, el acto de evaluación de A sobre B se convierte, a su vez, en una evaluación de A sobre la competencia transversal.

Para poder establecer una valoración en el dominio de la competencia por parte de un evaluador, se propone una fórmula que toma, como punto de partida, la calificación otorgada al evaluador, A, con la heteroevaluación y se modifica con la comparación entre las notas de la heteroevaluación y la coevaluación del evaluado, B. Todas las evaluaciones se hacen con el primer bloque de 6 preguntas, con su puntuación máxima de 17 puntos.

Los elementos de la fórmula son las siguientes:

- La nota otorgada a un estudiante o grupo evaluador, A, en la heteroevaluación, se denomina Nota1.

- La nota otorgada a un estudiante o grupo evaluado, B, en la heteroevaluación, se denomina Nota2H.

- La nota otorgada a un estudiante o grupo evaluado, B, por el estudiante o grupo evaluador, A, en la coevaluación, se denomina Nota2C.

- La diferencia entre los valores recibidos por la heteroevaluación y la coevaluación por el estudiante o grupo evaluado, B, se denomina Nota2 y se define como:

$$
\text { Nota } 2=\text { Nota } 2 H-N o t a 2 C
$$

La fórmula propuesta es una función definida a tramos, con la siguiente expresión:

$$
\left.\begin{array}{c}
\text { Si } \mid \text { Nota } \mid \leq 2 \rightarrow \text { Nota }=\operatorname{MiN}(\text { Nota } 1+(2-\mid \text { Nota } \mid), 17) \\
\text { Si } 3 \leq \mid \text { Nota } 2 \mid \leq 6 \rightarrow \text { Nota }=\text { Nota } 1-(\mid \text { Nota } 2 \mid-2) \\
\text { Si } \mid \text { Nota } 2 \mid \geq 7 \rightarrow \text { Nota }=\text { Nota } 1-6
\end{array}\right\}
$$

La puntuación que puede obtener un estudiante o grupo evaluador será de 0 a 17 puntos, pero puede no ser la misma con la que se partió inicialmente, sino que puede verse modificada de acuerdo a la calidad de la evaluación efectuada al otro estudiante o equipo. 
Las dos últimas preguntas sirven al profesorado como preguntas de control para validar el resultado obtenido y, sobre todo, para hacer visible si, en el caso de que el estudiante o equipo evaluador no partiera antes de la coevaluación de un nivel alto en el dominio de la competencia, ha mejorado su comprensión y asimilación de ésta después. Dado el carácter más subjetivo de esta apreciación, se deja al criterio del profesorado el que estas dos preguntas tengan mayor o menor influencia en la calificación final de la competencia.

Por último, para ajustar la calificación obtenida a la normativa de calificaciones de competencias transversales de la UPV, la cual contempla 4 niveles, A, B, C y D, de acuerdo con el nivel de dominio de la competencia alcanzado, se establece la siguiente correlación.

Nivel A (excelente) $\quad \Rightarrow 15 \leq$ Nota $\leq 17$

Nivel B (bien) $\quad \Rightarrow 12 \leq$ Nota $\leq 14$.

Nivel C (en desarrollo) $\Rightarrow 8 \leq$ Nota $\leq 11$.

Nivel D (no alcanzado) $\Rightarrow 0 \leq$ Nota $\leq 7$.

Los niveles más inferiores son más amplios que los superiores, ya que, las calificaciones que contienen implican un trabajo escaso en la competencia. 
Tabla 1. Lista de control para evaluación de la competencia específica en la experiencia sobre Aplicación y Pensamiento Práctico

1. Calidad de las instrucciones. Si son claras, detalladas correctas y no dejan pasos por describir.

Calificación (mínimo 0, máximo 2).

Justificar brevemente la calificación:

2. Modelo geométrico. Las carreras forman $90^{\circ}$ entre sí. La muñequilla de los pistones 2 y 4 está desfasada $180^{\circ}$ respecto la otra muñequilla. Si faltan piezas, se han creado adecuadamente.

Calificación (mínimo 0, máximo 2).

Justificar brevemente la calificación:

3. Procedimiento de construcción del modelo. Creación, copia, desplazamiento y giros de grupos y piezas.

Calificación (mínimo 0, máximo 2).

Justificar brevemente la calificación:

4. Programación del modelo. Creación de contadores Ang_Cig_720 de cada cilindro. Definición de polinomios, polin_1 y polin_2 de cada cilindro. Definición correcta de la fuerza en cada pistón.

Calificación (mínimo 0, máximo 2).

Justificar brevemente la calificación:

5. Funcionamiento en régimen permanente. Correcto funcionamiento, resultado coherente, comparación con el resultado del modelo propio del equipo evaluador.

Calificación (mínimo 0, máximo 2).

Justificar brevemente la calificación:

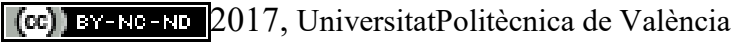

Congreso In-Red (2017) 
Tabla 2. Lista de control para la evaluación de la competencia transversal en Aplicación y Pensamiento Práctico.

Nombre:

Nombre:

Trabajo evaluado:

\begin{tabular}{|c|c|c|c|c|}
\hline & CRITERIOS & ¿Sí o no? & ¿En qué medida & \\
\hline 1 & $\begin{array}{l}\text { ¿Han entendido la tarea propuesta? ¿Lo explican de } \\
\text { forma adecuada en el trabajo? }\end{array}$ & $\begin{array}{l}\text { Sí } \square \\
\text { No } \square\end{array}$ & $\begin{array}{ll}\text { 1. } & \text { Inadecuado } \\
\text { 2. } & \text { Suficiente } \\
\text { 3. } & \text { Adecuado }\end{array}$ & $\begin{array}{l}\square \\
\square \\
\square\end{array}$ \\
\hline 2 & $\begin{array}{l}\text { ¿Han redactado un índice para el trabajo? ¿Resulta } \\
\text { adecuado? }\end{array}$ & $\begin{array}{l}\text { Sí } \square \\
\text { No } \square\end{array}$ & $\begin{array}{ll}\text { 1. } & \text { Inadecuado } \\
\text { 2. } & \text { Suficiente } \\
\text { 3. } & \text { Adecuado }\end{array}$ & $\begin{array}{l}\square \\
\square\end{array}$ \\
\hline 3 & $\begin{array}{l}\text { ¿Han interpretado los diagramas cinemáticos y de } \\
\text { encendido? }\end{array}$ & $\begin{array}{l}\text { Sí } \square \\
\text { No } \square\end{array}$ & $\begin{array}{ll}\text { 1. } & \text { Mal } \\
\text { 2. } & \text { Regular } \\
\text { 3. } & \text { Bien }\end{array}$ & $\begin{array}{l}\square \\
\square\end{array}$ \\
\hline 4 & $\begin{array}{l}\text { ¿Han introducido todas las partes del modelo } \\
\text { necesarias? }\end{array}$ & $\begin{array}{l}\text { Sí } \square \\
\text { No } \square\end{array}$ & $\begin{array}{l}\text { 1. No todas } \\
\text { 2. Todas }\end{array}$ & $\square$ \\
\hline 5 & $\begin{array}{l}\text { ¿Tienen claros los pasos a seguir para realizar el } \\
\text { trabajo? ¿Están bien enumerados? }\end{array}$ & $\begin{array}{l}\text { Sí } \square \\
\text { No } \square\end{array}$ & $\begin{array}{ll}\text { 1. } & \text { Mal } \\
\text { 2. } & \text { Regular } \\
\text { 3. } & \text { Bien }\end{array}$ & $\begin{array}{l}\square \\
\square\end{array}$ \\
\hline 6 & ¿Describen con precisión las operaciones a realizar? & $\begin{array}{l}\text { Sí } \square \\
\text { No } \square\end{array}$ & $\begin{array}{ll}\text { 1. } & \text { Mal } \\
\text { 2. } & \text { Regular } \\
\text { 3. } & \text { Bien }\end{array}$ & $\begin{array}{l}\square \\
\square \\
\square\end{array}$ \\
\hline 7 & ¿Difiere vuestro trabajo del que habéis evaluado? & $\begin{array}{l}\text { Sí } \square \\
\text { No } \square\end{array}$ & $\begin{array}{ll}\text { 1. } & \text { Poco } \\
\text { 2. } & \text { Bastante } \\
\text { 3. } & \text { Mucho }\end{array}$ & $\begin{array}{l}\square \\
\square \\
\square\end{array}$ \\
\hline 8 & $\begin{array}{l}\text { ¿Podríais mejorar vuestro trabajo después de haber } \\
\text { evaluado el del otro equipo? }\end{array}$ & $\begin{array}{l}\text { Sí } \square \\
\text { No } \square\end{array}$ & $\begin{array}{ll}\text { 1. } & \text { Poco } \\
\text { 2. } & \text { Bastante } \\
\text { 3. } & \text { Mucho }\end{array}$ & $\begin{array}{l}\square \\
\square \\
\square\end{array}$ \\
\hline
\end{tabular}




\section{Resultados}

\subsection{Diseño y Proyecto}

En este curso 2016-2017 se han evaluado 23 informes. En la Fig. 2 se muestra un histograma con las respuestas obtenidas en los diferentes apartados, y la evaluación de la competencia transversal. Se puede observar que los mejores resultados se obtienen en el apartado de Desarrollo, y los resultados más bajos en Discusión y Conclusiones. Esto nos indica que los estudiantes son capaces de planificar acciones adecuadas para resolver los objetivos del proyecto de forma eficiente, pero que tienen más dificultades a la hora de ser críticos con su propuesta, o de establecer formas para controlar el desarrollo de su plan.

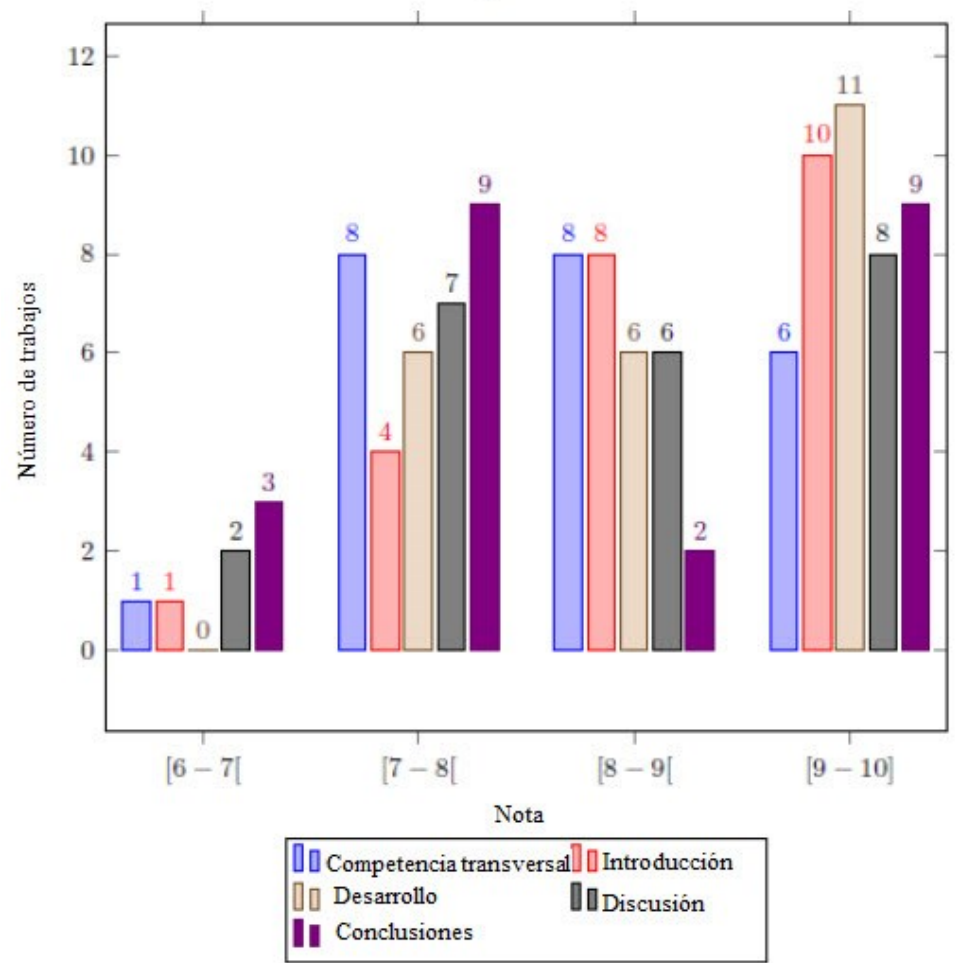

Fig. 2 Histograma que muestra las calificaciones de los diferentes apartados del trabajo, y de la competencia transversal, en una escala de 0 a 10.

\subsection{Compresión e integración}

En este curso 2016-2017 han participado 23 estudiantes y se han evaluado 11 trabajos. En la Fig. 3 se muestra una gráfica con las notas obtenidas, en la que se marca la línea que corresponde a la misma nota por parte del experto y de los pares, junto con las líneas que indican que hay una diferencia mayor de 3 puntos entre ambas.

(cc) EY-NC-ND 2017, UniversitatPolitècnica de València 
De los 23 estudiantes, sólo 19 realizaron correctamente la evaluación. Tres estudiantes no usaron la rúbrica correctamente y no pudieron ofrecer una nota razonable para los trabajos evaluados; otro estudiante no pudo evaluar porque hubo un error a la hora de asignar los trabajos a evaluar.

11 estudiantes realizaron una buena evaluación (diferencia menor de 3 puntos), mientras que 8 no lo hicieron, y su calificación se vió penalizada. Teniendo en cuenta este alto porcentaje de desviaciones, se considera que la evaluación por pares no es adecuada en esta actividad. Ello se debe a que para llevar a cabo una buena evaluación, los estudiantes deben tener un buen dominio de la asignatura. De forma que, como se pudo comprobar empíricamente, los estudiantes con buenas notas asignadas por el experto hicieron una buena evaluación de sus pares, pero los estudiantes con notas más bajas tuvieron mayores desviaciones respecto a esta referencia.

Aún así, se valora positivamente que la exposición de estos estudiantes con notas más bajas a los trabajos de otros compañeros puede mejorar su aprendizaje.

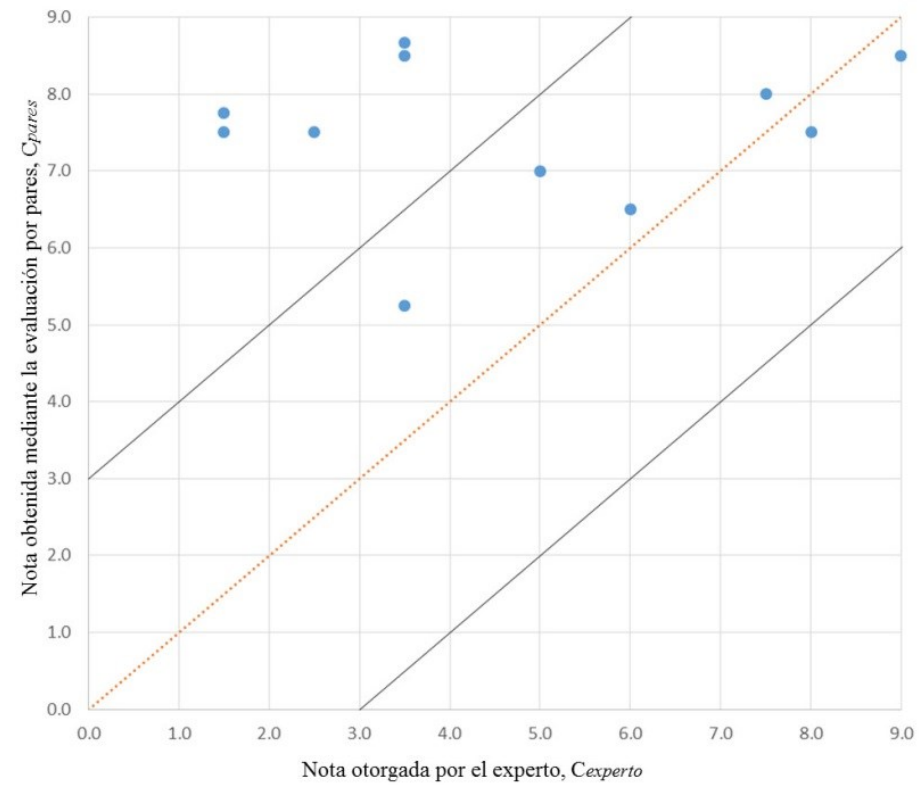

Fig. 3 Comparación entre las notas obtenidas por los diferentes trabajos por parte del experto y por parte de sus pares.

\subsection{Aplicación y Pensamiento Práctico}

En el desarrollo de la asignatura durante el curso 2015-2016, ya que no se dispone de los datos de este curso por ser una asignatura de segundo semestre, el número de coevaluaciones realizadas fue de 16 .

En la Tabla 3 se muestran las diferencias entre las valoraciones otorgadas en la heteroevaluación, es decir, por los profesores de la asignatura, en calidad de expertos, y en la coevaluación para la competencia específica. La variación se ha calculado como el valor absoluto de la diferencia entre la heteroevaluación y la coevaluación y normalizado sobre 10 puntos. Se opta por definir tres umbrales, los cuales se reflejan en la Tabla 3.

(cc) EY-NC-ND 2017, UniversitatPolitècnica de València 
Tabla 3. Variación entre valoraciones de heteroevaluación y coevaluación en la competencia específica.

\begin{tabular}{|c|c|c|c|}
\hline Variación & Menor o igual a 5\% & Entre 5\% y 10\% & Mayor que 10\% \\
\hline Núm. Evaluaciones & 11 & 4 & 1 \\
\hline \% Evaluaciones & 68,75 & 25,00 & 6,25 \\
\hline
\end{tabular}

Se puede apreciar que las valoraciones no difieren en exceso y que la poca presencia de casos con alta diferencia confirma la bondad del método.

En la Tabla 4 se muestran las diferencias entre las valoraciones otorgadas en la heteroevaluación y en la coevaluación para la competencia transversal. Los umbrales ahora se ajustan a los del procedimiento de calificación mostrado anteriormente.

Tabla 4. Variación entre valoraciones de heteroevaluación y coevaluación en la competencia transversal.

\begin{tabular}{|c|c|c|c|}
\hline Variación & Menor o igual a 2 p. & Entre 3 y 6 p. & Mayor que 7 p. \\
\hline Núm. Evaluaciones & 11 & 5 & 0 \\
\hline \% Evaluaciones & 68,75 & 31,25 & 0,00 \\
\hline
\end{tabular}

Los resultados son muy parecidos a los de la competencia específica.

La valoración del profesor de la competencia transversal, según los niveles marcados por la UPV son los siguientes.

Tabla 5. Valoración del profesor.

\begin{tabular}{|c|c|c|c|c|c|}
\hline Nivel & A & B & C & D & Total \\
\hline Núm. Equipos & 4 & 6 & 6 & 0 & 16 \\
\hline
\end{tabular}

La aplicación de la corrección en las calificaciones de los diferentes equipos según su coevaluación da como resultado la siguiente modificación:

Tabla 4. Modificación de la calificación.

\begin{tabular}{|c|c|c|c|c|c|}
\hline Nivel & A & B & C & D & Total \\
\hline Núm. Equipos & 5 & 4 & 5 & 2 & 16 \\
\hline
\end{tabular}

Se observan bastantes modificaciones en la calificación definitiva. Haciendo un estudio más detallado, se observó que los resultados modificados correspondían a valores 
fronterizos entre niveles y que la modificación venía a confirmar la tendencia que mostraban anteriormente.

Las dos últimas preguntas de la encuesta se consideraron de manera subjetiva, pero no aportaron información adicional a la que ya se había obtenido con el primer bloque y su puntuación.

\section{Conclusiones}

El desarrollo de actividades y herramientas de evaluación adecuadas es crítico para trabajar y evaluar competencias transversales. En este trabajo se han mostrado tres experiencias en las que se han enriquecido las actividades de evaluación empleadas anterioremente, para conseguir una experiencia de aprendizaje más completa por parte de los estudiantes.

El uso de la coevaluación en la evaluación de las competencias transversales es muy prometedor en cuanto a que añade valor formativo a la evaluación, y mejora la experiencia docente y de aprendizaje de los estudiantes. De las dos experiencias mostradas en esta comunicación, los resultados son valorados muy positivamente tanto por profesores como por los estudiantes en el caso de la competencia "Aplicación y Pensamiento Práctico". Las dificultades encontradas en la implementación de esta metodología para evaluar la competencia de "Compresión e Integración" sirven de aprendizaje para mejorar la propuesta en el próximo curso.

\section{Agradecimientos}

Los autores quieren mostrar su agradecimiento al Vicerrectorado de Estudios, Calidad y Acreditación de la Universitat Politècnica de València por la financiación obtenida a través del programa PIME 2016-17 bajo la referencia A26.

\section{Referencias}

ADAMS team at MSC Software. (2014). Adams Tutorial Kit for Mechanical Engineering Courses. MSC Software.

BOSTOCK, S. (2000). Student peer assessment. Learning Technology.

CARBALLEIRA, J., MARTÍNEZ-CASAS, J., SAHUQUILLO, O., SONSECA, A., DENIA, F.D., SUÑER, J.L., VILA, P., RÓDENAS, J.J., MARCO, O. (2015).

"Desarrollo de estrategias de evaluación de competencias transversales en asignaturas de ingeniería mecánica y de materiales.” En Congreso Nacional de Innovación Educativa y Docencia en Red de la Universitat Politècnica de València. (ISSN 978-849048-396-1). Valencia: Editorial UPV. 923-937.

CARBALLEIRA, J., MARTÍNEZ-CASAS, J., SAHUQUILLO, O., SONSECA, A., SUÑER, J.L., VILA, P., DENIA, F. D., RÓDENAS, J.J., MARCO, O. (2016). "Assessment of problem-solving skills and capacity for applying knowledge in practice 
in subjects related to mechanical and materials engineering" en Advances in Higher Education, Ed. Universitat Politècnia de València, Chapter 5, pp. 71-88.

CAVAS TOLEDO, M., CHICANO GARCÍA, F., LUNA VALERO, F., y MOLINA TANCO, L. (2011). "Autoevaluación y evaluación entre iguales en una asignatura de redes de ordenadores." En Jornadas de Enseñanza Universitaria de la Informática (17es: 2011: Sevilla).

LANGAN, M., y WHEATER, P. (2003). "Can students assess students effectively? Some insights into peer-assessment". Learning and Teaching in ACTION, 2 (1).

LIM, L.A. (2003). "Implementing effective peer assessment". CTDL Brief, 6 (3).

NULTY, D. (2008). "A Guide to Peer and Self Assessment: Approaches and Practice Strategies for Academics". Griffith Institute for Higher Education. Griffith University.

VILLA, A., y POBLETE, M. (2011). "Evaluación de competencias genéricas: principios, oportunidades y limitaciones". Bordón. Revista de pedagogía, 63(1), 147170.

UPV (2014). Proyecto institucional sobre Competencias Transversales. http://www.upv.es/contenidos/COMPTRAN/ [Consulta: 21 Marzo 2017] 